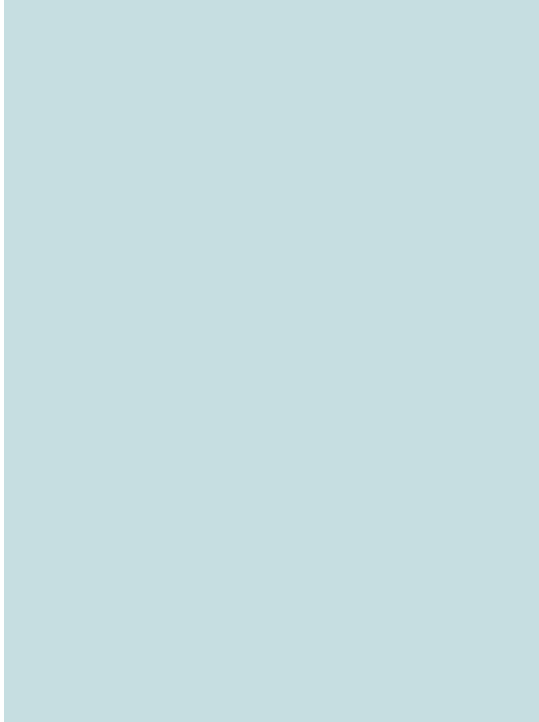

\title{
Carotid Artery Plaque
}

Morphology and Composition

in Relation to Incident

Cardiovascular Events: The

\section{Multi-Ethnic Study of Atherosclerosis}

$(\mathrm{MESA})^{1}$

Anna E. H. Zavodni, MD

Bruce A. Wasserman, MD

Robyn L. McClelland, PhD

Antoinette S. Gomes, MD

Aaron R. Folsom, MD, MPH

Joseph F. Polak, MD, MPH

João A. C. Lima, MD

David A. Bluemke, MD, PhD
${ }^{1}$ From the Department of Medical Imaging, Sunnybrook Health Sciences Centre, University of Toronto, Toronto, Ontario, Canada (A.E.H.Z.); Departments of Radiology (B.A.W., J.A.C.L.) and Medicine (J.A.C.L.), Johns Hopkins University, Baltimore, Md; Collaborative Health Studies Coordinating Center, University of Washington, Seattle, Wash (R.L.M.); Department of Radiology, University of California-Los Angeles School of Medicine, Los Angeles Calif (A.S.G.); Division of Epidemiology and Community Health, University of Minnesota, Minneapolis, Minn (A.R.F.); Department of Radiology, Tufts Medical Center, Boston, Mass (J.F.P.); and Department of Radiology and Imaging Sciences, National Institutes of Health Clinical Center, 10 Center Dr, Bldg 10/1C355, Bethesda, MD 20892 (D.A.B.). Received May 2, 2013; revision requested May 20; revision received December 3; final version accepted January 27, 2014. Address correspondence to D.A.B. (e-mail: david. bluemke@nih.gov).
Purpose:

Materials and Methods:

Results:

Conclusion:

The identification of vulnerable plaque characteristics with MR imaging aids in cardiovascular disease prediction and improves the reclassification of baseline cardiovascular risk.

${ }^{\circ}$ RSNA, 2014 
therosclerosis is a systemic disease process, and carotid artery imaging provides a useful surrogate marker of generalized vascular health. On the basis of autopsy studies, specific features of an atherosclerotic plaque represent unstable plaque that is prone to rupture (1-4). Features of vulnerable plaque include a lipid core with a thin fibrous cap and ulceration, and identification of a vulnerable plaque may serve as a marker with which to identify the "vulnerable patient” at risk for subsequent cardiovascular events $(5,6)$.

The carotid artery is highly accessible for imaging because of its relatively large size, superficial location, and relative immobility. Carotid ultrasonographic (US) intima-media thickness (IMT) has been shown to be a useful predictor of incident cardiovascular events (7-9). Magnetic resonance (MR) imaging is an alternative approach that can also be used to identify carotid plaque. Carotid MR imaging can accurately depict plaque components, such as the lipid core, and it can be used to identify and monitor vulnerable plaque (10-12). MR imaging has been used as a surrogate endpoint in pharmacologic intervention trials, and MR imaging depicts reduction in carotid wall area and plaque lipid content when lipid-lowering therapy is administered (12-14). Patients at high risk with symptomatic carotid artery disease have plaque features defined by MR imaging that are

\section{Advances in Knowledge}

- MR imaging of the carotid artery enables reliable identification of plaque composition in asymptomatic individuals.

- MR imaging remodeling index, lipid core, and calcium in the internal carotid artery are predictors of cardiovascular events.

- The combination of MR imaging remodeling index and presence of lipid core resulted in improvement of the net reclassification index compared with traditional risk factors for cardiovascular disease events. associated with cerebrovascular events $(15,16)$.

To our knowledge, a populationbased prospective study to determine if vulnerable plaque features add to risk for a cardiovascular event beyond traditional risk factors has not been performed previously. The hypothesis for this study was that imaging features of carotid plaque are independent predictors of cardiovascular risk in asymptomatic individuals. The purpose of this study was to determine if carotid plaque morphology and composition at MR imaging can be used to identify asymptomatic subjects at risk for cardiovascular events.

\section{Materials and Methods}

\section{Study Participants}

Institutional review boards at each site approved the study, and all sites were compliant with the Health Insurance Portability and Accountability Act. The authors had control of the data and information submitted for publication. Details of the Multi-Ethnic Study of Atherosclerosis (MESA) study design have been published previously (17). In brief, baseline recruitment was performed from July 2000 through September 2002 and yielded a total of 6814 adults aged $45-84$ years. All participants were free of cardiovascular disease at enrollment, and they were stratified according to sex and race or ethnicity at six different field centers (Baltimore, Md; Chicago, Ill; Forsyth County, NC; Los Angeles, Calif; New York, NY; and St Paul, Minn). A full list of participating MESA investigators

\section{Implications for Patient Care}

- Key imaging features of carotid MR imaging studies include remodeling of the carotid artery wall and composition of the arterial wall.

- Identification of a lipid core in patients with carotid plaques may enable identification of individuals who are at risk for subsequent cardiovascular events. and institutions is available online (http://www.mesa-nhlbi.org). A total of 6624 participants underwent carotid US and common and internal carotid IMT measurement. We used a sampling scheme with stratification according to IMT status to enroll approximately 1000 subjects for carotid MR imaging: A total of 600 participants drawn from all six centers were recruited from the group of individuals within the MESA cohort who were at or above the 85th percentile according to carotid IMT. An additional 400 subjects from the Baltimore and Los Angeles centers were sampled from the group below the 85th percentile according to internal carotid artery (ICA) IMT.

\section{Cardiovascular Risk Factors}

Centrally trained clinical teams blinded to participant outcome collected information on cardiovascular risk factors that composed the Framingham risk score during standardized clinic visits. The history and physical examination findings obtained during the second MESA clinic visit were used in this analysis. Smoking history was assessed

Published online before print

10.1148/radiol.14131020 Content code: CA

Radiology 2014; 271:381-389

Abbreviations

$\mathrm{CCA}=$ common carotid artery

ICA = internal carotid artery

IMT = intima-media thickness

MESA = Multi-Ethnic Study of Atherosclerosis

$\mathrm{NRI}=$ net reclassification improvement

\section{Author contributions:}

Guarantor of integrity of entire study, D.A.B.; study concepts/study design or data acquisition or data analysis/interpretation, all authors; manuscript drafting or manuscript revision for important intellectual content, all authors; approval of final version of submitted manuscript, all authors; literature research, A.E.H.Z., B.A.W., A.S.G., J.F.P., J.A.C.L., D.A.B.; clinical studies, A.S.G., J.F.P., D.A.B.; statistical analysis, A.E.H.Z., B.A.W., R.L.M.; and manuscript editing, A.E.H.Z., B.A.W., A.S.G., A.R.F., J.F.P., J.A.C.L., D.A.B.

\section{Funding:}

This research was supported by the National Institutes of Health (grants R01-HL-69905 and N01-HC-95159 through N01-HC-95168).

Conflicts of interest are listed at the end of this article. 
via self-report and categorized as current, any cigarette smoking within the past 30 days, or none. Blood pressure was measured via the right arm after participants were seated comfortably for 5 minutes. Three measurements were obtained with an automated oscillometric sphygmomanometer (Dinamap Pro 1000; Critikon, Tampa, Fla). The average of the last two measurements was used for analysis.

At baseline, after an overnight fast, blood was drawn with a minimally traumatic venipuncture for inflammatory marker measurements, including a high-sensitivity assay for C-reactive protein and interleukin 6 . Within 30 days after carotid MR imaging, a fasting blood test was performed to measure glucose, hemoglobin $A_{1 c}$, total plasma, and low- and high-density lipoprotein cholesterol levels. Diabetes was considered present if the current glucose level or any prior fasting glucose level was above $125 \mathrm{mg} / \mathrm{dL}(6.9 \mathrm{mmol} / \mathrm{L})$ or if a participant was using hypoglycemic medication.

\section{Carotid IMT}

US carotid artery IMT measurements in MESA have been described previously (18). This examination included an assessment of the ICA that extended from the bulb to $1 \mathrm{~cm}$ above the carotid sinus. The near and far walls were examined for maximum wall thickening, and this segment was recorded from three different angles (anterior oblique, lateral, and posterior oblique). The common carotid artery (CCA) was imaged over a 1-cm length in a segment just below the common carotid bulb (18). Reproducibility was assessed by replicating measurements in 66 participants; the interclass correlation coefficient for the replicated readings was 0.84 .

\section{Carotid MR Imaging}

The carotid MR imaging protocol has been described previously (Table 1) (19). Each center acquired carotid MR images with a 1.5-T imager and use of dedicated carotid coils. Five electrocardiographically gated T1- and T2-weighted fat-suppressed inversion recovery black-blood fast spin-echo images were

\begin{tabular}{|c|c|c|c|}
\hline \multicolumn{4}{|c|}{ MR Imaging Sequence Parameters for Black-Blood Images } \\
\hline Parameter & $\begin{array}{l}\text { Proton Density-weighted } \\
\text { Long-Axis View }\end{array}$ & $\begin{array}{l}\text { Transverse } \\
\text { T2-weighted View }\end{array}$ & $\begin{array}{l}\text { Transverse T1-weighted View } \\
\text { before and after Gadolinium } \\
\text { Enhancement }\end{array}$ \\
\hline Repetition time & 2 RR intervals & 2 RR intervals & $1 \mathrm{RR}$ interval \\
\hline Echo time (msec) & 5 & 68 & 5 \\
\hline Inversion time (msec) & 600 & 600 & 350 \\
\hline Section thickness (mm) & 2 & 2 & 2 \\
\hline Section gap & 0 & 0 & 0 \\
\hline Matrix & $256 \times 256$ & $256 \times 256$ & $256 \times 256$ \\
\hline Field of view $(\mathrm{cm})$ & 14 & 14 & 14 \\
\hline Echo train length & 10 & 10 & 10 \\
\hline No. of signals acquired & 1 & 1 & 1 \\
\hline Bandwidth (kHz) & 62.5 & 62.5 & 62.5 \\
\hline
\end{tabular}

Note.-Images were cardiac gated, with repetition time based on the RR interval of the cardiac cycle. Inversion time was set automatically and based on heart rate to minimize blood pool signal on the basis of estimated.

obtained through the internal carotid artery (ICA). All ICA sections were positioned perpendicular to the area of greatest wall thickness. One transverse section of the common carotid artery was obtained $1 \mathrm{~cm}$ below the carotid bifurcation. Intravenous contrast material (0.2 mL per kilogram of body weight, Omniscan; GE Healthcare, Princeton, NJ) was administered in consenting participants. After 5 minutes, T1weighted imaging was repeated at the same locations (Figs 1, 2).

Image analysis was conducted at the Johns Hopkins MR imaging core laboratory. Two analysts contoured the CCA and ICA images with the largest lipid core area or the thickest wall area if no lipid core was present. The analysis methods for MR imaging were described previously (19). An experienced physician (D.A.B., 20 years of cardiovascular MR imaging experience) supervised the process. The adventitial contour and lumen area were used to compute the wall area and mean wall thickness. Plaque components, including lipid core, calcification, and ulceration, were contoured with subsequent automatic detection and segmentation of the overlying fibrous cap. Classification of plaque components was performed as previously reported, in which the lipid core showed low signal intensity on gadolinium-enhanced T1-weighted images (20). Calcification showed very low signal intensity as a subcomponent of the atherosclerotic plaque. Reproducibility was assessed by replicating measurements for $10 \%$ of the participants 3 months after the initial evaluation. The intraclass correlation coefficient for interobserver reproducibility was $98 \%$ for the adventitial contour, $99 \%$ for lumen area, $88 \%$ for wall area, and $86 \%$ for mean wall thickness.

\section{Event Assessment}

MESA event ascertainment has been described previously (21). In brief, MESA-defined cardiovascular disease includes a composite of coronary heart disease events, such as (a) fatal coronary heart disease, nonfatal myocardial infarction, cardiac arrest, and angina (definite and probable when associated with coronary revascularization); (b) cerebrovascular disease; (c) fatal and nonfatal stroke; and (d) other cardiovascular death. Records were successfully obtained for $98 \%$ of reported cardiovascular events that required hospitalization.

\section{Statistical Analysis}

An initial weighted multivariable Cox proportional hazard model was created by using age, sex, systolic blood 


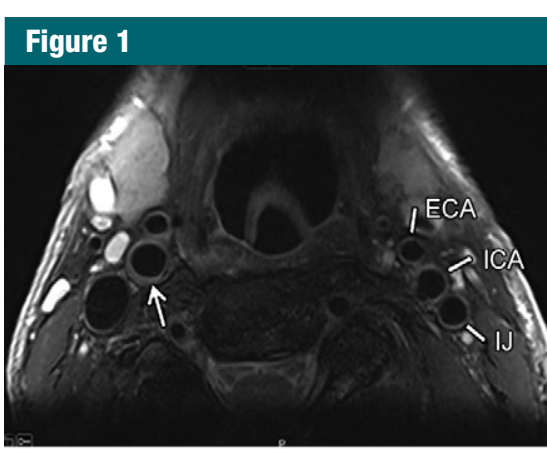

a.
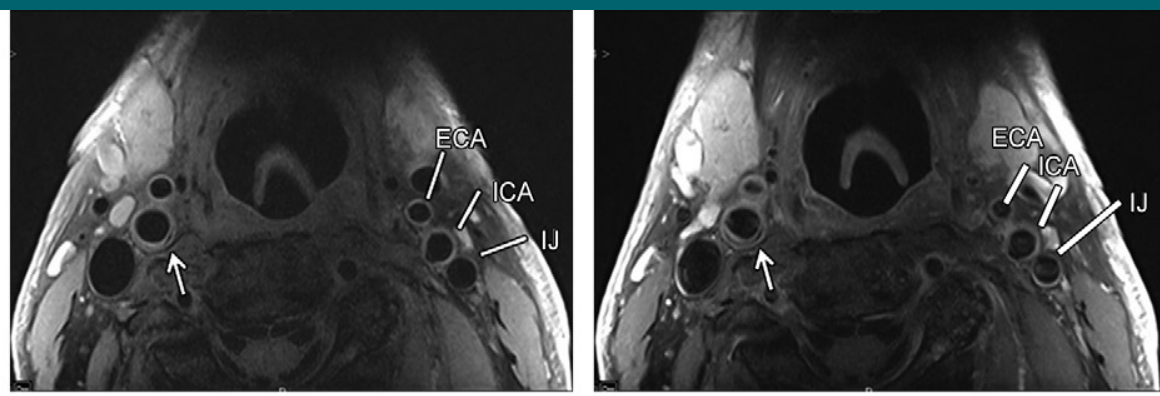

b.

c.

Figure 1: Transverse (a) T2-weighted, (b) unenhanced T1-weighted, and (c) gadolinium-enhanced T1-weighted MR images in an 82-year-old man. Pulse sequence parameters are described in Table 1. The left ICA wall shows no plaque or significant wall thickening. The right ICA shows plaque with a lipid core (arrow). $E C A=$ external carotid artery, $I J=$ internal jugular vein.

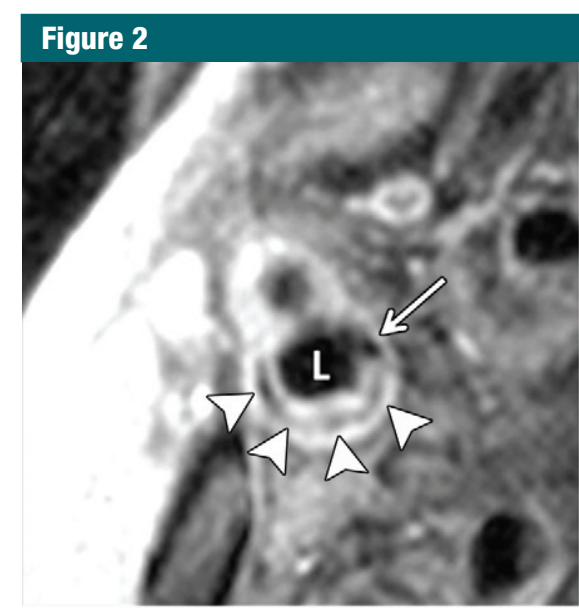

a.

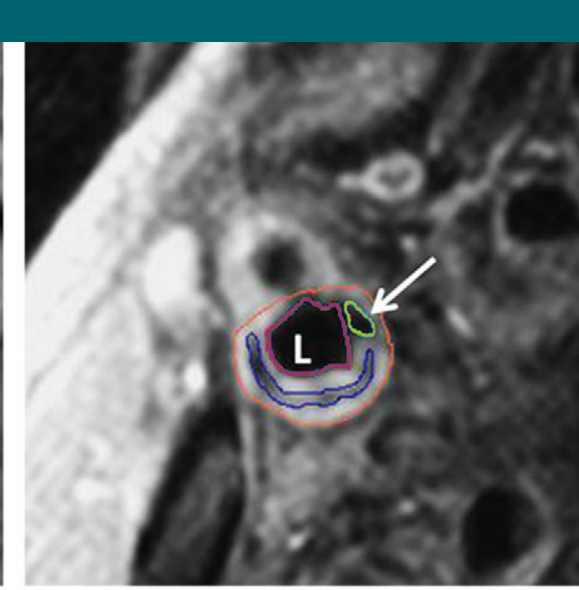

b.

Figure 2: Transverse gadolinium-enhanced T1-weighted MR images obtained superior to the carotid artery bifurcation in a 72-year-old man. $L=$ ICA lumen. (a) Low-signal-intensity calcium (arrow) and lipid core (arrowheads) can be seen. (b) Note contouring of the ICA. The outer adventitial wall (red), lipid core (blue), calcification (green), and vessel lumen (purple) are visible.

pressure, antihypertensive medication use, total cholesterol level, highdensity lipoprotein cholesterol level, diabetes, and smoking status data $(22,23)$. Additional weighted models were generated by separately adding to the base model of the IMT and MR imaging predictors of cardiovascular disease for both the internal and common carotid arteries. The MR imaging predictors included remodeling index (wall area divided by the sum of wall area and lumen area) (24) for both the ICA and the CCA and lipid core presence for the ICA (Fig 3). The remodeling index normalized the wall area to the total vessel area. Higher values of the remodeling index indicate greater atherosclerotic burden independent of vessel size $(24,25)$. Because of the low prevalence of a lipid core within the CCA and the low prevalence of calcification and ulceration within either the ICA or the CCA, these features were not included in the statistical models.

Sensitivity analysis was performed after excluding plaques less than 1.5 $\mathrm{mm}$ in maximum thickness to ensure that spatial resolution in the detection lipid core was not a limiting factor. Further analysis was performed by stratifying for race and adding covariates to control for body size (height, height squared, or body mass index), low-density lipoprotein cholesterollowering or lipid-lowering medication use, and hemoglobin $A_{1 c}$ and inflammatory marker levels (C-reactive protein or interleukin 6). Height may be an important covariate to adjust for native vessel size (19), and use of lipid-lowering medication can result in regression of lipid-rich atherosclerotic plaque (12). Hemoglobin $A_{1 c}$ level was included as a covariate, as previous associations between hemoglobin $A_{1 c}$ level and ICA and CCA IMTs have been demonstrated, even in the absence of clinically evident diabetes (26). Further adjustment was made for the baseline interleukin 6 level because elevated values have been associated with events independent of carotid IMT in high-risk patients (27).

The $C$ statistic for the base and each imaging model was generated. For the IMT and MR imaging models with the largest $C$ statistic, the net reclassification improvement (NRI) was calculated (28). The NRI for imaging features was determined after recalibrating the baseline risk model as a 5.5-year risk score with categories parallel to the current National Cholesterol Education Program guidelines and defined as low $(0 \%$ to $<1 \%$ per year), intermediate $(1 \%-2 \%$ per year $)$, or high $(>2 \%$ per year) risk (22).

All statistical analysis was performed by using statistical software 
Figure 3

Internal Carotid

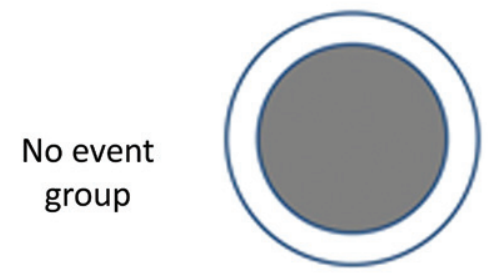

Lumen $46 \mathrm{~mm}^{2}$ Wall area $29 \mathrm{~mm}^{2}$

Remodeling index 0.39

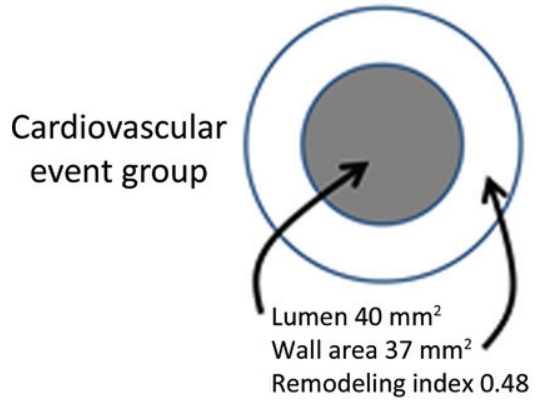

Common Carotid

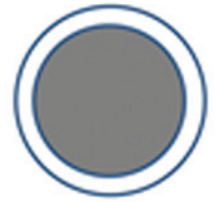

Lumen $37 \mathrm{~mm}^{2}$ Wall area $26 \mathrm{~mm}^{2}$ Remodeling index 0.41

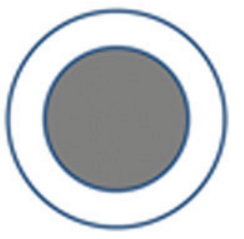

Lumen $46 \mathrm{~mm}^{2}$ Wall area $29 \mathrm{~mm}^{2}$ Remodeling index 0.42

Figure 3: Diagram shows representative changes in carotid wall area, lumen area, and remodeling index for the ICA and CCA. Representative values of lumen and wall areas are taken from Table 1. The normal remodeling index tends to be approximately 0.4 independent of vessel size. The remodeling index increases with relatively increased atherosclerotic burden. Diagrams are not to scale.

(SAS Enterprise Guide, version 9.2; SAS Institute, Cary, NC). A two-sided $P$ value of less than .05 was considered to indicate a significant difference.

\section{Results}

\section{Participant Characteristics}

Table 1 summarizes participant characteristics at the time of carotid MR imaging by event category. The group that experienced an event was characterized by older age, a higher prevalence of male subjects, a higher proportion of white and Hispanic participants, higher systolic blood pressure, a greater proportion of participants taking antihypertensive and lipid-lowering medication, and more subjects with diabetes. A total of 946 participants had evaluable carotid MR images. MR imaging lipid core was present in $19.1 \%$ of participants. Calcium and ulceration were seen in $2.4 \%$ and $0.2 \%$ of the studies, respectively. None of the study subjects had high-grade carotid artery stenosis.

One participant was older than 79 years (no Framingham risk score), and six participants were missing one or more of the Framingham risk score covariates. Over a mean follow-up period of 5.5 years \pm 1.2 (standard deviation), 59 (6.3\%) of $939 \mathrm{MR}$ imaging participants experienced an incident cardiovascular event (Table 1). Most events were related to coronary atherosclerosis: nineteen participants experienced a myocardial infarction, 22 experienced angina (definite angina, $n=21$; probable angina followed by revascularization, $n=1$ ), four died from coronary heart disease, and one participant experienced resuscitated cardiac arrest. Cerebrovascular disease was the incident event in nine participants, and four other participants died from cerebrovascular disease.

For the CCA, the mean IMT was $0.96 \mathrm{~mm}$, and the MR imaging mean wall thickness was $1.04 \mathrm{~mm}$ (Pearson correlation coefficient, 0.50; $P<.001$ ). The MR imaging CCA outer wall area was $63.7 \mathrm{~mm}^{2}$, the lumen area was 37.6 $\mathrm{mm}^{2}$, the wall area was $26.0 \mathrm{~mm}^{2}$, and the remodeling ratio was 0.41 . A lipid core was seen in $0.1 \%$ of the weighted studies, and no calcium or ulceration was identified.

Table 2 summarizes imaging parameters according to vessel and cardiovascular events. For both the ICA and the CCA, US-measured IMT and MR imaging mean wall thickness and wall area were greater in participants with cardiovascular events. For the ICA alone, greater MR imaging remodeling index and presence of lipid core or calcium were associated with more cardiovascular events. For CCA alone, a larger MR imaging outer contour area was associated with events.

\section{Carotid Artery Characteristics as a Predictor of Cardiovascular Events}

Multivariable Cox proportional hazards models are displayed in Table 3 . The base model included the Framingham risk factor score, Subsequent models included one or more imaging features. The ICA US IMT, MR imaging remodeling index, and MR imaging lipid core presence were all associated with cardiovascular events that had similar hazard ratios-a one-standard-deviation increase-ranging from 2.17 (US IMT) to 2.85 (MR imaging remodeling index). These hazard ratios were higher for the ICA than for the CCA for all imaging variables.

The $C$ statistics for the US and MR imaging variables are shown in Table 3 . Traditional risk factors (represented by the Framingham risk score) were predictive of cardiovascular disease events with a $C$ statistic of 0.696 (95\% confidence interval: $0.616,0.775)$. The most predictive model consisted of the combination of MR imaging-defined ICA remodeling index and lipid core presence with a $C$ statistic of 0.734 (95\% confidence interval: $0.652,0.817)$. As displayed in Tables 4 and 5 , baseline risk factors were refit to produce a 5.5year risk score for a common set of 470 subjects, each of whom had undergone carotid IMT, CCA, and ICA MR imaging 


\section{Table 2}

\section{Description of Study Participants according to Incident Event Status during Follow-up with Results Weighted to Reflect the Baseline MESA Cohort}

\begin{tabular}{|c|c|c|c|}
\hline Risk Factor & No Event & Event & $P$ Value \\
\hline \multicolumn{4}{|l|}{ Characteristic } \\
\hline Age $(y)^{\star}$ & $62.6 \pm 25.5$ & $68.1 \pm 20.9$ & $<.001^{\dagger}$ \\
\hline Male sex (\%) & 49.8 & 68.6 & $<.001^{\dagger}$ \\
\hline \multicolumn{4}{|l|}{ Race $(\%)$} \\
\hline African American & 24.5 & 8.6 & $<.001^{\dagger}$ \\
\hline White & 33.4 & 55.3 & $\ldots$ \\
\hline Asian & 24.3 & 7.5 & $\ldots$ \\
\hline Hispanic & 17.8 & 28.7 & $\ldots$ \\
\hline \multicolumn{4}{|l|}{ Body size } \\
\hline Weight $(\mathrm{kg})^{\star}$ & $76.0 \pm 42.8$ & $78.5 \pm 29.4$ & .33 \\
\hline Height $(m)^{\star}$ & $1.66 \pm 0.25$ & $1.67 \pm 0.19$ & .41 \\
\hline Body mass index $\left(\mathrm{kg} / \mathrm{m}^{2}\right)^{\star}$ & $27.4 \pm 12.4$ & $27.9 \pm 8.6$ & .45 \\
\hline \multicolumn{4}{|l|}{ Blood pressure } \\
\hline Systolic blood pressure $(\mathrm{mmHg})^{\star}$ & $121 \pm 50$ & $131 \pm 51$ & $.002^{\dagger}$ \\
\hline Antihypertensive medication use (\%) & 36.5 & 65.0 & $<.001^{\dagger}$ \\
\hline \multicolumn{4}{|l|}{ Cholesterol and lipids } \\
\hline Total cholesterol level $(\mathrm{mg} / \mathrm{dL})^{\star \star}$ & $190 \pm 86$ & $199 \pm 104$ & .10 \\
\hline Low-density lipoprotein level $(\mathrm{mg} / \mathrm{dL})^{\star \neq}$ & $113 \pm 77$ & $123 \pm 100$ & $.04^{\dagger}$ \\
\hline High-density lipoprotein level $(\mathrm{mg} / \mathrm{dL})^{\star \ddagger}$ & $51 \pm 36$ & $47 \pm 26$ & .07 \\
\hline Lipid-lowering medication use (\%) & 22.1 & 37.8 & $<.001^{\dagger}$ \\
\hline \multicolumn{4}{|l|}{ Diabetes } \\
\hline Yes $(\%)$ & 12.3 & 35.6 & $<.001^{\dagger}$ \\
\hline Hemoglobin $A_{11}$ level $(\%)^{\star \S}$ & $5.7 \pm 2.8$ & $6.9 \pm 5.8$ & $<.001^{\dagger}$ \\
\hline Smoking history (\%) & 9.6 & 11.7 & .18 \\
\hline \multicolumn{4}{|l|}{ Inflammatory markers } \\
\hline C-reactive protein level $(\mathrm{mg} / \mathrm{L})^{\star \star \|}$ & $0.5 \pm 2.9$ & $0.8 \pm 2.1$ & .08 \\
\hline Interleukin 6 level $(\mathrm{pg} / \mathrm{mL})^{*}$ & $0.1 \pm 1.6$ & $0.3 \pm 1.1$ & .06 \\
\hline
\end{tabular}

* Data are mean \pm standard deviation.

${ }^{\dagger}$ Difference was significant $(P<.05)$.

¥ To convert to SI units (millimoles per liter), multiply by 0.0259 .

$\S$ To convert to SI units (proportion of total hemoglobin), multiply by 0.01 .

" To convert to SI units (nanomoles per liter), multiply by 9.524 .

and complete risk factor assessment. The NRI was significant with inclusion of the MR imaging ICA remodeling index and lipid core presence (NRI was $7.4 \%$ for participants without cardiovascular events and $15.8 \%$ for participants with cardiovascular events; $P=$ .02); however, it was not significant for the ICA or CCA MR imaging remodeling index alone or the US IMT.

Sensitivity analysis performed after we excluded study subjects with a maximal plaque thickness less than 1.5 mm yielded comparable results: adding race, height, height squared or BMI, use of low-density lipoprotein cholesterollowering or lipid-lowering medication, hemoglobin $A_{1 \mathrm{c}}$ level, and C-reactive protein or interleukin 6 level yielded imaging parameter estimates of similar magnitude and significance. Similar results were achieved when individual FRS components were substituted for the composite score.

\section{Discussion}

In this study, we examined the use of carotid MR imaging to predict cardiovascular events in an asymptomatic population. MR imaging has shown great potential to noninvasively define substructural components of atherosclerotic plaque. In particular, a lipid core has long been suggested as one of several atherosclerosis features related to plaque vulnerability for rupture (1-4). Our results support the concept that adverse carotid arterial remodeling and a lipid core at MR imaging confer increased risk for subsequent cardiovascular events in asymptomatic individuals. Since cerebrovascular disease was not a common event in this study, we conclude that the lipid core enabled identification of a "vulnerable patient" rather than a vulnerable plaque $(5,6)$. Indeed, about $16 \%$ more subjects with events and 7\% of subjects without events were correctly reclassified compared with traditional risk factors when both the carotid remodeling index and the lipid core were used for risk stratification.

A consensus panel $(5,6)$ recommended the term vulnerable plaque to identify thrombosis-prone plaques and plaques that are likely to progress rapidly. One patient may have multiple vulnerable plaques in different arterial beds. Largely on the basis of autopsy studies (1-4), the vulnerable plaque was noted to have active inflammation, a thin cap with a large lipid core, fissuring of the plaque surface, stenosis of more than $90 \%$, or some combination thereof. Vulnerable plaques frequently are nonstenotic. The same consensus panel noted that outcome studies, such as the current study, are needed to validate the use of the various imaging markers and biomarkers of a vulnerable plaque $(5,6)$.

One other prospective outcome study was performed to evaluate the importance of plaque morphology with MR imaging in asymptomatic subjects. Takaya et al (29) followed 154 asymptomatic patients with relatively highgrade $50 \%-70 \%$ carotid stenosis for a mean of 38 months. Twelve ipsilateral cerebrovascular events occurred in the 38-month follow-up period. Their results showed that thinned or ruptured plaque, intraplaque hemorrhage size, lipid core size, and maximum wall thickness were associated with subsequent events (29).

The current study was conducted in the context of an epidemiologic study of 


\section{Table 3}

\section{US IMT and Carotid MR Imaging Parameters Compared with Events}

\begin{tabular}{|c|c|c|c|c|c|c|}
\hline \multirow[b]{2}{*}{ Risk Factor } & \multicolumn{3}{|c|}{ Internal Carotid Artery } & \multicolumn{3}{|c|}{ Common Carotid Artery } \\
\hline & No Event & Event & $P$ Value & No Event & Event & $P$ Value \\
\hline IMT thickness at US (mm) & $1.05 \pm 1.46$ & $1.62 \pm 1.33$ & $<.001^{\star}$ & $0.95 \pm 0.31$ & $1.07 \pm 0.33$ & $<.001^{*}$ \\
\hline \multicolumn{7}{|l|}{ MR imaging } \\
\hline Thickness (mm) & $1.08 \pm 0.98$ & $1.41 \pm 1.15$ & $<.001^{\star}$ & $1.03 \pm 0.29$ & $1.13 \pm 0.34$ & $.004^{\star}$ \\
\hline Outer contour area $\left(\mathrm{mm}^{2}\right)$ & $76.0 \pm 64.2$ & $77.3 \pm 57.5$ & .75 & $63.2 \pm 22.4$ & $69.5 \pm 28.0$ & $.01^{\star}$ \\
\hline Lumen area $\left(\mathrm{mm}^{2}\right)$ & $46.5 \pm 48.0$ & $40.4 \pm 42.1$ & $.04^{\star}$ & $37.5 \pm 14.7$ & $40.2 \pm 18.6$ & .11 \\
\hline Wall area $\left(\mathrm{mm}^{2}\right)$ & $29.4 \pm 31.0$ & $36.9 \pm 32.5$ & $.002^{\star}$ & $25.7 \pm 10.3$ & $29.4 \pm 12.4$ & $.002^{*}$ \\
\hline Remodeling index & $0.39 \pm 0.27$ & $0.48 \pm 0.30$ & $<.001^{\star}$ & $0.41 \pm 0.08$ & $0.42 \pm 0.08$ & .09 \\
\hline Lipid core present (\%) & 17.8 & 47.9 & $<.001^{\star}$ & 0.1 & 0 & .72 \\
\hline Calcium present (\%) & 1.7 & 19.5 & $<.001^{\star}$ & 0 & 0 & $\ldots$ \\
\hline Ulcer present (\%) & 0.2 & 0 & .46 & 0 & 0 & $\ldots$ \\
\hline
\end{tabular}

Note.-Unless otherwise indicated, data are mean \pm standard deviation.

* Difference was significant $(P<.05)$.

\section{Table 4}

\section{Multivariable Cox Proportional Hazards Models of Carotid Artery MR Imaging and IMT} Predictors of Cardiovascular Disease

\begin{tabular}{|c|c|c|c|c|}
\hline Model & Hazard Ratio* & $P$ Value & C Statistic ${ }^{\dagger}$ & $P$ Value \\
\hline Framingham risk score alone & Reference & .. & $0.696(0.616,0.775)$ & \\
\hline \multicolumn{5}{|l|}{ Framingham risk score and ICA } \\
\hline MR imaging remodeling index alone & $2.85(1.36,5.97)$ & $.006^{\ddagger}$ & $0.720(0.639,0.801)$ & .34 \\
\hline MR imaging remodeling index and & $2.15(0.89,5.19)$ & .09 & $0.734(0.652,0.817)$ & .16 \\
\hline lipid core ${ }^{\S}$ & $1.77(0.76,4.14)$ & .18 & & \\
\hline US IMT & $2.17(1.30,3.63)$ & $.003^{\ddagger}$ & $0.724(0.652,0.795)$ & .08 \\
\hline \multicolumn{5}{|l|}{ Framingham risk score and CCA } \\
\hline MR imaging remodeling index & $1.76(1.10,2.83)$ & $.02^{\ddagger}$ & $0.711(0.632,0.789)$ & .57 \\
\hline US IMT & $1.52(0.97,2.37)$ & .07 & $0.707(0.628,0.787)$ & .51 \\
\hline
\end{tabular}

Note.-Framingham risk score encompasses age, sex, systolic blood pressure, antihypertensive medication use, total cholesterol and high-density lipoprotein levels, diabetes (present or absent), and smoking status (current or not). Remodeling index is calculated by dividing carotid artery wall area by adventitial contour area.

* Data in parentheses represent a one standard deviation increase.

${ }^{\dagger}$ Data in parentheses are $95 \%$ confidence intervals.

‡ Difference was significant $(P<.05)$ compared with traditional risk factors, as represented by the Framingham risk score cardiovascular disease events defined in Table 1

$\S$ There is a separate hazard ratio for both the remodeling index and the lipid core when used together in the model.

individuals who were asymptomatic at baseline enrollment. Study participants were followed prospectively, and there was abundant information available for risk stratification in the study subjects. Plaque features, such as plaque ulceration, thin fibrous cap, and intraplaque hemorrhage, were rare in this asymptomatic group; therefore, they were not included in the statistical analysis. We found that the combination of wall thickness and lipid core presence resulted in a significant improvement as assessed by the net reclassification index for prediction of cardiovascular events compared with traditional risk factors. Of note, however, the overall $C$ statistic showed only modest improvement over traditional risk factors, with an increase from 0.70 to 0.73 . Our design provides greater assurance than do small studies of symptomatic patients that the observed imaging risk factors are markers of subclinical atherosclerosis rather than the effects of the event process itself or subsequent medical intervention.

Unlike prior studies, our results indicate a more generalized relationship between carotid plaque severity and cardiovascular events. As summarized by Madjid et al (30), previous autopsy series have shown that most cases of fatal coronary thrombosis result from one plaque rupture; however, most individuals have multiple unstable lesions at the time of this event. Thus, plaque growth, destabilization, and repair may be a generalized process in certain individuals who are at high risk.

The Framingham Offspring Cohort study (9) suggested that ICA IMT offers a modest incremental value in $C$ statistic to the Framingham risk score in the prediction of cardiovascular events; a nonsignificant improvement in the $C$ statistic was seen in this study. While the NRI for IMT was of similar magnitude as that in the Framingham Offspring cohort, it did not reach significance in our study. The nonsignificant improvement in the $C$ statistic and NRI was likely related to differences in sample size (2946 participants in the Framingham Offspring Cohort study vs 939 subjects in the MESA carotid MR imaging cohort) and a slightly 


\section{Table 5}

Reclassification of the Baseline Risk Factors Model after Addition of ICA MR Imaging or IMT Predictors of Cardiovascular Disease for Both the ICA and the CCA

\begin{tabular}{|c|c|c|c|c|c|c|c|c|c|}
\hline & \multicolumn{4}{|c|}{ No Event $(n=432)$} & \multicolumn{4}{|c|}{ Event $(n=38)$} & \multirow[b]{2}{*}{$P$ Value } \\
\hline & Increase & No Change & Decrease & Change (\%) & Increase & No Change & Decrease & Change (\%) & \\
\hline \multicolumn{10}{|l|}{ ICA } \\
\hline MR imaging remodeling index & 57 & 300 & 75 & 4.2 & 7 & 27 & 4 & 7.8 & .19 \\
\hline $\begin{array}{l}\text { MR imaging remodeling index } \\
\text { and lipid core }\end{array}$ & 65 & 270 & 97 & 7.4 & 10 & 24 & 4 & 15.8 & $.02^{*}$ \\
\hline US & 41 & 328 & 63 & 5.1 & 6 & 29 & 3 & 7.8 & .12 \\
\hline \multicolumn{10}{|l|}{ CCA } \\
\hline MR imaging remodeling index & 35 & 335 & 62 & 6.2 & 4 & 30 & 4 & 0 & .42 \\
\hline US & 29 & 363 & 40 & 2.5 & 3 & 33 & 2 & 2.6 & .40 \\
\hline
\end{tabular}

Note.-Unless otherwise indicated, data are numbers of subjects. Framingham risk score was calculated for a 5.5-year risk according to National Cholesterol Education Program guidelines and defined as low risk ( $0 \%$ to $<1 \%$ per year), intermediate risk ( $1 \%-2 \%$ per year), or high risk ( $>2 \%$ per year).

${ }^{\star}$ Difference was significant $(P<.05)$.

shorter follow-up interval $(7.2$ years vs 5.5 years).

Calculation of the NRI helps gauge the effects of adding new risk factors to the traditional Framingham risk factors for marginal improvements in the $C$ statistic (28). We assessed the NRI for three clinically relevant risk categories (23) that were chosen to reflect clinical practice and the recent National Cholesterol Education Program Adult Treatment Panel III guidelines (22). A limitation of this method is that these categories remain somewhat arbitrary from a biologic standpoint, and small shifts in these cut points may produce large increases in NRI (31). In addition, a limitation of any observational cohort study is that event-free participants have not experienced an event since the most recent follow-up period; however, they may in fact experience the endpoint a short time later. Since our models were refit for a 5.5-year follow-up period (as opposed to the 10-year follow-up period for which the Framingham risk score is calculated [32]), this short follow-up could have reduced the discriminatory ability of our test and decreased the overall power of our observations. With a low incidence of events and a sampling strategy of including a greater proportion of individuals with the thickest ICA IMT values, our sampling scheme biases the NRI results in favor of a test that can be used to correctly down-classify high-risk individuals who do not experience an event.

Availability and cost-effectiveness are important determinants of whether carotid MR imaging is used clinically in cardiovascular risk adjustment and screening. Black-blood sequences for plaque morphology could be readily added to existing carotid MR angiography protocols for clinical purposes. Our results suggest that there is a robust relationship between carotid MR imaging plaque and lipid core and subsequent incident cardiovascular events. MR imaging has been previously shown to be more reproducible then US $(33,34)$. Our results and others further show that MR imaging features can be used to predict cardiovascular endpoints and thus bolster the use of MR imaging as a surrogate marker of efficacy in therapeutic studies $(13,35)$.

In conclusion, the identification of vulnerable plaque with MR imaging maximizes cardiovascular disease prediction and significantly improves the reclassification of baseline cardiovascular risk. Carotid artery remodeling and lipid core presence measured noninvasively with MR imaging are independent predictors of new cardiovascular events in individuals without a history of cardiovascular disease.
Acknowledgments: The authors thank the other investigators, staff, and participants in the MESA study for their valuable contributions. They also thank Amersham for supplying the contrast material.

Disclosures of Conflicts of Interest: A.E.H.Z. No relevant conflicts of interest to disclose. B.A.W. Financial activities related to the pre sent article: none to disclose. Financial activities not related to the present article: has a patent pending (no. 13/922,111). Other relationships: none to disclose. R.L.M. No relevant conflicts of interest to disclose. A.S.G. No relevant conflicts of interest to disclose. A.R.F. No relevant conflicts of interest to disclose. J.F.P. No relevant conflicts of interest to disclose. J.A.C.L. No relevant conflicts of interest to disclose. D.A.B. No relevant conflicts of interest to disclose.

\section{References}

1. Davies MJ. Anatomic features in victims of sudden coronary death. coronary artery pathology. Circulation 1992;85(1,Suppl):I19I24.

2. Davies MJ, Richardson PD, Woolf N, Katz DR, Mann J. Risk of thrombosis in human atherosclerotic plaques: role of extracellular lipid, macrophage, and smooth muscle cell content. Br Heart J 1993;69(5):377-381.

3. Farb A, Burke AP, Tang AL, et al. Coronary plaque erosion without rupture into a lipid core. a frequent cause of coronary thrombosis in sudden coronary death. Circulation 1996;93(7):1354-1363.

4. Richardson PD, Davies MJ, Born GV. Influence of plaque configuration and stress distribution on fissuring of coronary atherosclerotic plaques. Lancet 1989;2(8669):941-944. 
5. Naghavi M, Libby P, Falk E, et al. From vulnerable plaque to vulnerable patient: a call for new definitions and risk assessment strategies: part II. Circulation 2003;108(15):17721778.

6. Naghavi M, Libby P, Falk E, et al. From vulnerable plaque to vulnerable patient: a call for new definitions and risk assessment strategies: part I. Circulation 2003;108(14):1664-1672.

7. Chambless LE, Heiss G, Folsom AR, et al. Association of coronary heart disease incidence with carotid arterial wall thickness and major risk factors: the Atherosclerosis Risk in Communities (ARIC) Study, 19871993. Am J Epidemiol 1997;146(6):483494.

8. O'Leary DH, Polak JF, Kronmal RA, Manolio TA, Burke GL, Wolfson SK Jr. Carotidartery intima and media thickness as a risk factor for myocardial infarction and stroke in older adults. Cardiovascular Health Study Collaborative Research Group. N Engl J Med 1999;340(1):14-22.

9. Polak JF, Pencina MJ, Pencina KM, O'Donnell CJ, Wolf PA, D'Agostino RB Sr. Carotid-wall intima-media thickness and cardiovascular events. N Engl J Med 2011;365(3):213-221.

10. Cai J, Hatsukami TS, Ferguson MS, et al. In vivo quantitative measurement of intact fibrous cap and lipid-rich necrotic core size in atherosclerotic carotid plaque: comparison of high-resolution, contrast-enhanced magnetic resonance imaging and histology. Circulation 2005;112(22):3437-3444

11. Ayaori M, Momiyama Y, Fayad ZA, et al. Effect of bezafibrate therapy on atherosclerotic aortic plaques detected by MRI in dyslipidemic patients with hypertriglyceridemia. Atherosclerosis 2008;196(1):425-433.

12. Helft G, Worthley SG, Fuster V, et al. Progression and regression of atherosclerotic lesions: monitoring with serial noninvasive magnetic resonance imaging. Circulation 2002;105(8):993-998.

13. Fayad ZA, Mani V, Woodward M, et al. Safety and efficacy of dalcetrapib on atherosclerotic disease using novel non-invasive multimodality imaging (dal-PLAQUE): a randomised clinical trial. Lancet 2011;378(9802):1547-1559.

14. Zhao XQ, Yuan C, Hatsukami TS, et al. Effects of prolonged intensive lipid-lowering therapy on the characteristics of carotid atherosclerotic plaques in vivo by MRI: a case-control study. Arterioscler Thromb Vasc Biol 2001;21(10):1623-1629.

15. Altaf N, Daniels L, Morgan PS, et al. Detection of intraplaque hemorrhage by magnetic resonance imaging in symptomatic patients with mild to moderate carotid stenosis predicts recurrent neurological events. J Vasc Surg 2008;47(2):337-342.

16. Turc G, Oppenheim C, Naggara O, et al. Relationships between recent intraplaque hemorrhage and stroke risk factors in patients with carotid stenosis: the HIRISC study. Arterioscler Thromb Vasc Biol 2012;32(2):492-499.

17. Bild DE, Bluemke DA, Burke GL, et al. Multi-ethnic study of atherosclerosis: objectives and design. Am J Epidemiol 2002;156(9):871-881.

18. Polak JF, Wong Q, Johnson WC, et al. Associations of cardiovascular risk factors, carotid intima-media thickness and left ventricular mass with inter-adventitial diameters of the common carotid artery: the Multi-Ethnic Study of Atherosclerosis (MESA). Atherosclerosis 2011;218(2):344349 .

19. Wasserman BA, Sharrett AR, Lai S, et al. Risk factor associations with the presence of a lipid core in carotid plaque of asymptomatic individuals using high-resolution MRI: the multi-ethnic study of atherosclerosis (MESA). Stroke 2008;39(2):329-335.

20. Wasserman BA, Smith WI, Trout HH 3rd, Cannon RO 3rd, Balaban RS, Arai AE. Carotid artery atherosclerosis: in vivo morphologic characterization with gadolinium-enhanced double-oblique MR imaging-initial results. Radiology 2002;223(2):566-573.

21. Bluemke DA, Kronmal RA, Lima JA, et al. The relationship of left ventricular mass and geometry to incident cardiovascular events: the MESA (Multi-Ethnic Study of Atherosclerosis) study. J Am Coll Cardiol 2008;52(25):2148-2155.

22. Expert Panel on Detection, Evaluation, and Treatment of High Blood Cholesterol in Adults. Executive summary of the third report of the National Cholesterol Education Program (NCEP) expert panel on detection, evaluation, and treatment of high blood cholesterol in adults (Adult Treatment Panel III). JAMA $2001 ; 285(19): 2486-2497$.

23. D’Agostino RB Sr, Vasan RS, Pencina MJ, et al. General cardiovascular risk profile for use in primary care: the Framingham Heart Study. Circulation 2008;117(6):743-753.

24. Kerwin W, Xu D, Liu F, et al. Magnetic resonance imaging of carotid atherosclerosis: plaque analysis. Top Magn Reson Imaging 2007;18(5):371-378.

25. Saam T, Yuan C, Chu B, et al. Predictors of carotid atherosclerotic plaque progression as measured by noninvasive mag- netic resonance imaging. Atherosclerosis 2007;194(2):e34-e42.

26. McNeely MJ, McClelland RL, Bild DE, et al. The association between A1C and subclinical cardiovascular disease: the multi-ethnic study of atherosclerosis. Diabetes Care 2009;32(9):1727-1733.

27. Okazaki S, Furukado S, Abe Y, et al. Association of inflammatory markers and carotid intima-media thickness with the risk of cardiovascular events in high-risk patients. Cerebrovasc Dis 2010;30(2):180187.

28. Pencina MJ, D’Agostino RB Sr, D’Agostino RB Jr, Vasan RS. Evaluating the added predictive ability of a new marker: from area under the ROC curve to reclassification and beyond. Stat Med 2008;27(2):157-172; discussion 207-212.

29. Takaya N, Yuan C, Chu B, et al. Association between carotid plaque characteristics and subsequent ischemic cerebrovascular events: a prospective assessment with MRI-initial results. Stroke 2006;37(3):818-823.

30. Madjid M, Zarrabi A, Litovsky S, Willerson JT, Casscells W. Finding vulnerable atherosclerotic plaques: is it worth the effort? Arterioscler Thromb Vasc Biol 2004;24(10):1775-1782.

31. Ware JH, Cai T. Comments on 'evaluating the added predictive ability of a new marker: from area under the ROC curve to reclassification and beyond' by MJ Pencina et al, Statistics in Medicine (DOI: 10.1002/ sim.2929). Stat Med 2008;27(2):185-187.

32. Wilson PW, D'Agostino RB, Levy D, Belanger AM, Silbershatz $H$, Kannel WB. Prediction of coronary heart disease using risk factor categories. Circulation 1998;97(18):1837-1847.

33. Duivenvoorden R, de Groot E, Elsen BM, et al. In vivo quantification of carotid artery wall dimensions: 3.0-Tesla MRI versus Bmode ultrasound imaging. Circ Cardiovasc Imaging 2009;2(3):235-242.

34. Harloff A, Zech T, Frydrychowicz A, et al. Carotid intima-media thickness and distensibility measured by MRI at $3 \mathrm{~T}$ versus high-resolution ultrasound. Eur Radiol 2009;19(6):1470-1479.

35. Corti R, Fuster V, Fayad ZA, et al. Effects of aggressive versus conventional lipid-lowering therapy by simvastatin on human atherosclerotic lesions: a prospective, randomized, double-blind trial with high-resolution magnetic resonance imaging. J Am Coll Cardiol 2005;46(1):106-112. 